\title{
Big data technology in construction
}

\author{
Nikolay Garyaev* and Venera Garyaeva \\ Moscow State University of Civil Engineering, Yaroslavskoe shosse, 26, Moscow, 129337, Russia
}

\begin{abstract}
The article presents the results of the analysis of the use of large amounts of data in the construction industry, new trends such as BIM, IOT, cloud computing, intelligent buildings and smart cities with great prospects for application. These problems are related to the presence of huge amounts of data produced by the construction industry during the entire life cycle of a building, which are not fully used for optimizing processes and making decisions in construction.
\end{abstract}

\section{Introduction}

The development of information technology leads to the constant growth of structured and unstructured data volumes (Big Data). Today, companies have to deal with petabytes of data. Google processes over 30 petabytes of data per day, while Facebook receives more than 10 million photos per hour. Presumably, by 2020 the amount of data on the Internet will increase to 44 zettabytes. This avalanche-like data growth allows you to solve a huge number of problems and create new research directions. Today, Big Data is compared to the oil of our time. The availability of big data allows you to take a fresh look at various areas of human activity. The construction industry is no exception. It is also significantly affected by the digital revolution.

\section{Information modeling}

One of such areas is Building Information Modeling (BIM), designed to collect multidimensional information about a construction site, with the support of interdisciplinary cooperation of stakeholders. BIM data is usually compressed in various proprietary formats in $3 \mathrm{D}$ coding. These various data collected in the BIM model are constantly updated in the process of design, construction and operation and are saved after the end of the service life of the objects. BIM files of a building model easily reach $100 \mathrm{~GB}$ in size. This data in any format and form is of great value for the industry.

With the advent of embedded devices and sensors, objects begin to generate massive data at the stage of operation and maintenance, which ultimately leads to a sharp increase in data on the construction site. This huge amount of BIM data is pushing the construction industry into the era of big data. Big data has certain parameters, first, their size (terabytes, petabytes, data exabytes, etc.); secondly, a variety of heterogeneous data formats, such as text, audio, video, graphics, data from sensors and much more; and third, the transfer speed.

\footnotetext{
* Corresponding author: garyaev@mgsu.ru
} 
Construction data is huge due to large volumes of design data, graphs, corporate resource planning system (ERP), financial data, etc. A variety of construction data can be classified into various file formats supported in construction programs and applications. In addition, construction data is dynamic, which stems from the streaming nature of data sources, such as sensors, RFID and BMS (building management system). Using this data to optimize the design, construction and operation of the next frontier of innovation in the industry.

Today, the tasks of developing methods for estimating parameters of big data, defining approaches to their structuring, accumulation, testing and storage, as well as determining the relationship between formats and their streaming become very important. The competitive advantage is the ability to transform the results of big data analysis to identify, understand and respond to hidden trends in order to make management decisions.

In the field of big data analysis, there are many areas, but in a simplified form, they are usually divided into two categories: Big Data engineering and Big Data Analytics (Scientist). Although these directions are interdependent, they differ from each other.

\section{Big Data engineering}

Big Data engineering is engaged in designing a system for processing, collecting and storing data that allows processing petabytes of data and providing access to various user applications to the results of data processing. This requires specialists with good programming skills, knowledgeable network technologies, able to interact via the Internet and work professionally with computer equipment.

\section{Big Data Analytics}

Big Data Analytics searches for patterns in large data arrays obtained from off-the-shelf systems developed by Big Data engineering. The direction of data analysis in itself is quite extensive and includes such specializations as Data Mining, Text Mining, Visual Mining, OLAP, Process Mining, Web Mining, Real-Time Data Mining, Stream Mining, Multimedia Mining, Spatiotemporal Data Mining, Information Network Analysis, Biological Data Mining, Financial Data Mining

Big data analysis includes the development of various systems of classification and forecasting to study trends and patterns, followed by the interpretation of results.

To work with data analysis requires specialists who own the methods of search, selection, grouping, analysis, integration and visualization of data.

One of the Big Data processing tools available is the open source Apache Hadoop and Apache Spark platforms, which allow you to process huge amounts of semi-structured data from heterogeneous sources. Platforms allow you to analyze data lake of data websites, financial analysis, research, create microservice buses for real-time data processing, for example, site segmentation, fraud detection, transport monitoring, etc.

However, studies using large building data processing are rarely used to process data from BIM building information models, because the data is intertwined and very relative and difficult to distribute across different Hadoop blocks. This placement leads to a decrease in query performance due to the increase in disk I / O needed to merge sparse data together for analysis. To overcome this, it is necessary to develop a preprocessing and data sharing module that allows you to analyze and split the logically relevant portions of BIM data (by floor number or material family), while storing them in adjacent Hadoop locations. To maximize the use of the central processor, it is necessary to introduce multithreading during data analysis. 
Based on the above, you can configure Hadoop for BIM data, and implement query components as applications for collision detection and estimating the number of information models. The purpose of the study is to develop an interactive user interface for querying data, based on natural language processing to extract very complex BIM data.

Big Data Analytics has rich intellectual traditions borrowed from a wide variety of areas of expertise. Traditionally, many related disciplines have the same goal - to find useful patterns in the data. These related areas include statistics, data mining, predictive analytics, business analytics, knowledge discovery from data, data analytics, Data Science and now Big Data. Big data analysis includes many of the methods that have already been developed. Effective analysis begins with properly configured data collection, allowing you to formulate reliable conclusions, so most of the work presented focuses on the analysis of big data.

\section{Cloud computing Big Data}

Cloud computing is an Internet computing paradigm where access to demand is provided to the shared pool of customizable resources. The idea of the approach is to transfer data storage and perform calculations to third-party data centers.

As a result, it becomes possible to simultaneously access cloud services to several users without the need to purchase separate licenses. Cloud services offer three service options. The first is infrastructure as a service (IaaS). In IaaS, the user is granted access to manage virtual computers and cloud network services. The second is a platform as a service (PaaS). PaaS provides the user with services related, in particular, to development environments, such as operating systems, programming languages or databases. The third service is the provision of software as a service (SaaS). SaaS provides access to applications, such as Revit 360, over the Internet. Cloud computing is widely used in the construction industry because it supports the integration of tasks in BIM applications. The author used the cloud for context-sensitive computing based on BIM. Were connected Google SketchUp, Google Apps, Autodesk BIM 360 and Viewpoint, in the Amazon EC2 cloud to support most of the functions of the designers. An attempt was made to integrate cloud computing with the latest technologies such as VR / AR and social networking business services, creating a virtual environment for better visualization and understanding of BIM models. Cloud computing can significantly accelerate the introduction of IT in the construction industry, transforming many specific tasks in the design, construction and operation of buildings. The role of big data in this transformation is huge.

\section{Big Data and the Internet of Things (IOT)}

The rapidly developing technologies lead to the fact that electronic devices are becoming smaller and more powerful, and high-speed Internet is becoming cheaper and more accessible. This has led to the emergence of various devices connected to the Internet and ultimately, the emergence of the Internet of Things (IOT) technology. The main idea is to integrate smart devices and vital control objects through the Internet. Combining physical and digital worlds creates exciting opportunities. IOT has been successfully applied in all industries, including logistics, transportation, asset tracking, smart homes, smart buildings, energy, defense and agriculture. The research conducted by the author showed the possibility of using RFID to monitor construction and quality control, and, in the case of integration with BIM documents, to quickly search and locate objects. The use of IOT in construction represents an unlimited number of use cases and requires thousands of sensory 
devices for data collection, which necessitates working with big data. Figure 1 shows the scheme of data transmission over the Internet.

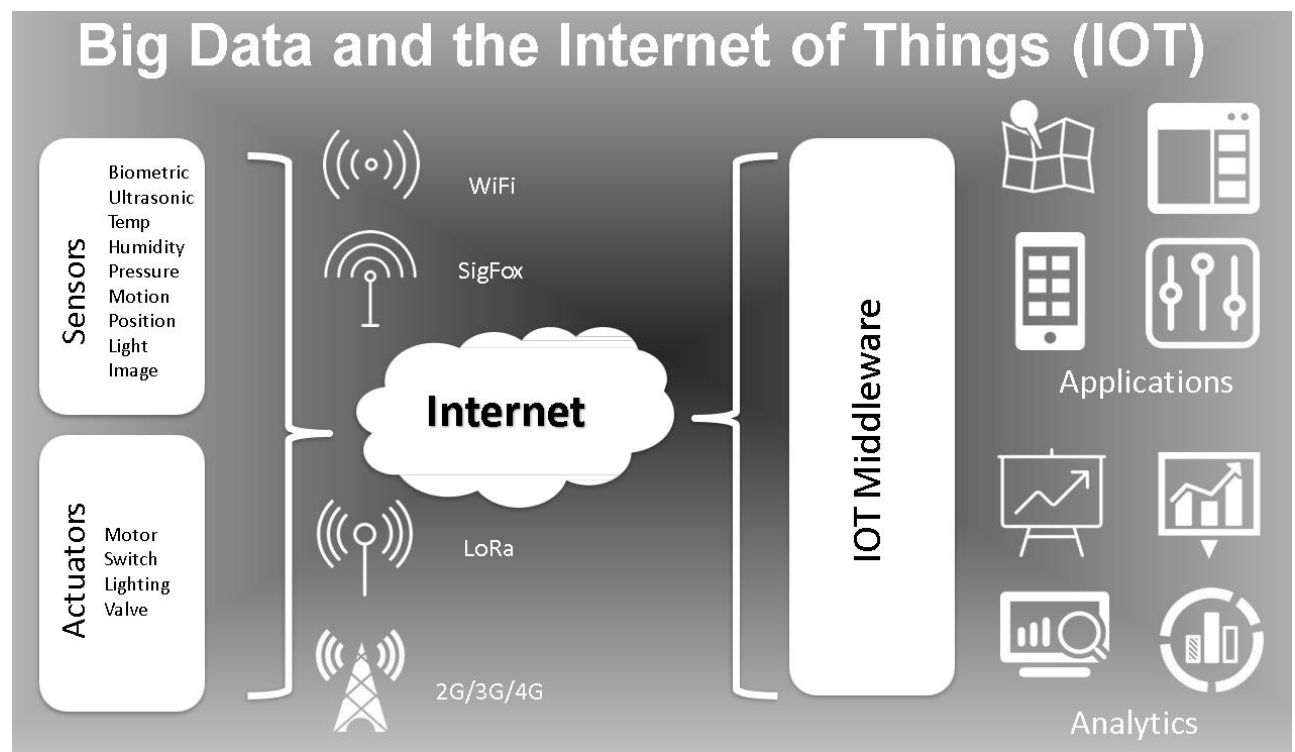

Fig. 1. Big Data and the Internet of Things (IOT)

\section{Smart buildings and Big Data}

With the development of digital technology, a modern building is becoming a complex intellectual system that includes such subsystems as building automation, life safety, telecommunications, user systems, object management systems, control and interaction with building maintenance services. Smart building integrates technology into building systems through a single view. These systems generate huge amounts of data, and most of this data often remains unused. To realize the full potential of a smart building and optimize the overall performance of a building using predictive analytics, a serious analysis of big data is necessary. For example, an approximate calculation of the database volumes obtained from the operation of sensors related to temperature, airflow, lighting, movement of people, air quality control installed in a 24-story building, showed that over 8 months more than 2 billion data records will be accumulated that reaches the limits of conventional relational databases. To overcome this limitation is possible only on the basis of the implementation of Big Data technology, which will make up for the technological gap and ensure the integration of sensors, users, control systems, equipment to expand the innovative services provided by the intelligent building.

\section{Smart City and Big Data}

Solving the problems of the Smart City to improve the functioning of urban services based on big data analysis opens up new perspectives for developers and data analysts. Scopes of the Big Data for the Smart city have a wide range of application. The distribution of video surveillance cameras in cities is widely used for various kinds of analysts and is successfully used to combat crime. Figure 2 presents an ecosystem of big data and a smart city. 


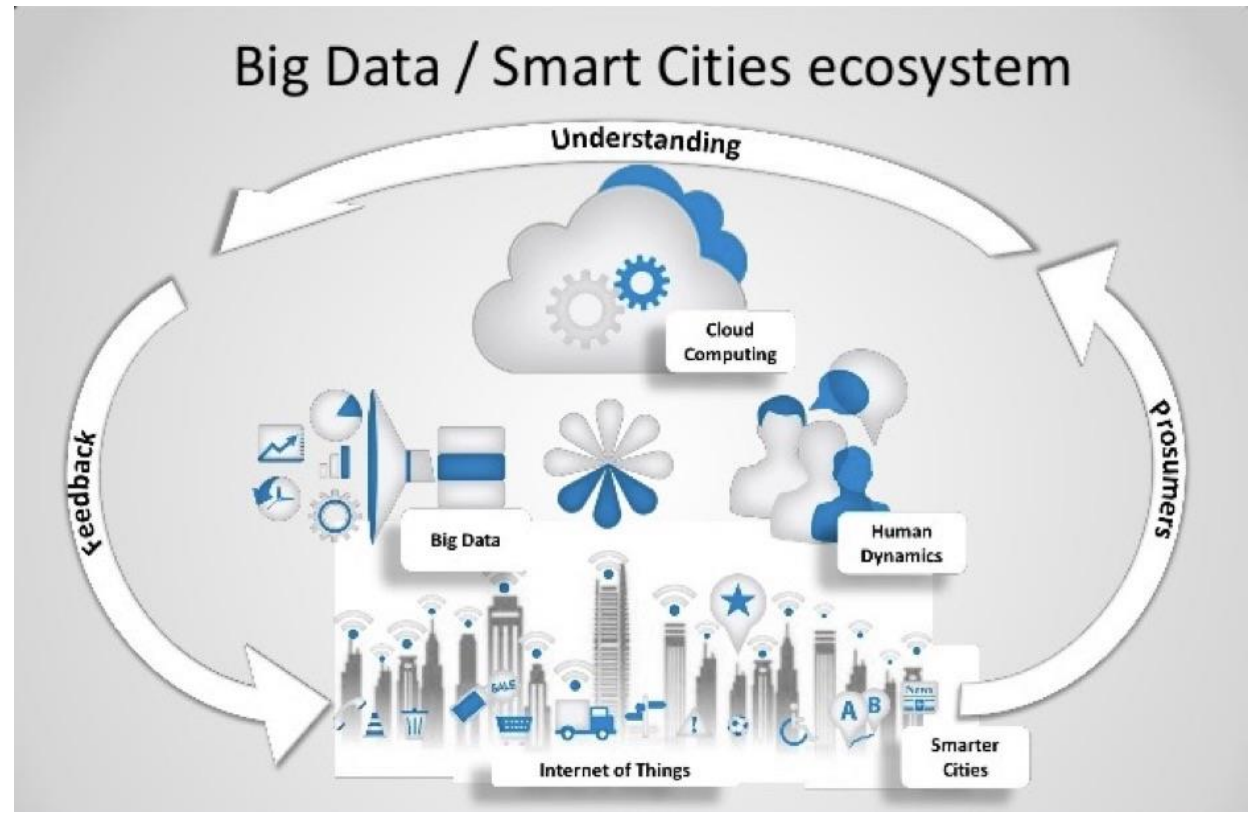

Fig. 2. Smart City and Big Data

An important task is to analyze traffic for event detection (event detection), which can greatly affect the traffic situation in the city. For planning urban infrastructure, it is important to track the geolocation of urban events that attract a large number of people, such as sports events, festivals, concerts, etc. for their planning and conduct.

\section{Conclusions}

The use of big data technology in construction is not enough. The huge amounts of data produced by the construction industry during the entire life cycle of a building are not fully utilized. At the same time, the development of technologies required for storing, computing, processing, analyzing and visualizing big data demonstrates an increase in the efficiency of construction processes.

The article analyzes the use of big data technologies in the industry, discusses the possibilities and ways of using big data in cloud computing, the Internet of things, smart buildings, smart city. As a result of the study, we concluded that the introduction of the latest, very flexible and powerful big data technology is slow, despite the fact that the analyst based on this data has long been used in the construction industry. The demand for Big Data technology is increasing with the introduction of new trends in construction practice, such as BIM, IOT, cloud computing, intelligent buildings and smart cities that have great potential for use.

This work was financially supported by Ministry of Science and Higher Education of the Russian Federation (\#NSh-3492.2018.8).

\section{References}

1. Ginzburg A., Shilova L., Adamtsevich A., Shilov L. Implementation of BIMtechnologies in Russian construction industry according to the international experience / Journal of Applied Engineering Science, 14 (4), p. 457-460 (2016) 
2. Garyaev N.A. Design and analysis of information model hotel complex. MATEC Web of Conferences 2016. C. 06007.

3. Garyaev N.A. Geographically distributed learning network construction industry. Computing in Civil and Building Engineering Proceedings 2014 International Conference. 2014. C. 1578-1585.

4. Garyaev P.N. Computer-aided zoning and urban planning. Computing in Civil and Building Engineering Proceedings 2014 International Conference. 2014. C. 1618-1625.

5. Garyaeva V.V., Garyaev N.A. Integrated assessment of the technical condition of the housing projects on the basis of computer technology. Computing in Civil and Building Engineering Proceedings 2014 International Conference. 2014. C. 1336-1343.

6. Ginzburg A. Sustainable Building Life Cycle Design / 15th International Conference on Topical Problems of Architecture, Civil Engineering, Energy Efficiency and Ecology, TPACEE-2016; Tyumen State University of Architecture and Civil Engineering: MATEC Web of Conferences, Volume 73, 02018 (2016)

7. Kachanov S., Ginzburg A. Methodology for building automated systems for monitoring engineering (load-bearing) structures, and natural hazards to ensure comprehensive safety of buildings and constructions / International Journal of Applied Engineering Research ISSN 0973-4562 Volume 11, Number 3 (2016), Research India Publications, 2016, -pp 1660-1665.

8. Kagan P.B., Kulikov V.G., Information modeling of urban planning development, Applied Mechanics and Materials Vols., - pp.951-954 (2013)

9. Muminova S.R., Kagan P.B., BIM training course in construction university, Proceedings of the 11th International Conference on Construction Applications of Virtual Reality, pp. $72-77$ (2011)

10. Ryzhkova A., Ginzburg A. Information system of risks analysis and management for construction projects with energy-efficient technologies in use / International Journal of Applied Engineering Research ISSN 0973-4562 Volume 10, Number 21 (2015), Research India Publications, 2015, -pp 41828-41830. 